\title{
SPACETIME AS A CAUSAL SET: UNIVERSE AS A GROWING BLOCK?
}

\begin{abstract}
The causal set programme towards a quantum theory of gravity is situated vis-à-vis the long-standing debate between eternalism (block theory) and past-presentism or possibilism (growing block theory) in the philosophy of time. It is argued that despite "appearances" and declarations to the contrary, the programme does not side with growing block theorists when it comes to harboring a robust notion of Becoming - at least, not more than familiar relativistic theories on continuous spacetime manifolds. The problem stems mainly from the postulate of discrete general covariance - a requirement imposed upon the only fully worked out kind of dynamics for causal sets to date, a dynamics of a classical stochastic process.
\end{abstract}

\section{Introduction}

The philosophy of time has long been troubled by the (at least, apparent) inhospitality of spacetime physics towards a robust notion of Becoming. The representation of a spacetime and of its material contents by means of 4-dimensional differentiable manifold hosting a family of geometric object fields seems biased $a b$ initio in favor of the eternalist metaphysical thesis that Donald Williams ([1951] 1967) has dubbed "the doctrine of the manifold". Of course, some philosophers have tried to salvage a notion of Becoming, even in the less hospitable environments of relativistic worlds, either by weakening the philosophical notion (e.g., by localizing the present) or by dismantling some of the physics (e.g., by introducing a preferred inertial frame). Still, till recently, theory construction in physics has proceeded without paying much attention to incorporating a mechanism for realizing genuine Becoming.

A significant exception to this ethos in theoretical physics is the causal set programme (CSP) in quantum gravity, which made its first official appearance in 1987 (Bombelli et al. 1987). The CSP has two core postulates concerning the deep structure of spacetime: that it is discrete and that it can be interpreted with reference to causal concepts and principles. ${ }^{1}$ In more detail, a spacetime as envisioned by classical general relativity is just a "coarse" approximation to a causal set, a locally finite partially ordered set. From a philosophical perspective, the CSP may be regarded as an outgrowth of the causal theories of time or

1 Dowker $(2005 ; 2006)$ and Wallden $(2010)$ are three exceptionally helpful overviews. 
spacetime - and, arguably, an outgrowth that overcomes significant hurdles of antecedent lines of attack. ${ }^{2}$ Hence one could claim that the CSP deserves more attention by philosophers than the little it has attracted so far (Stachel 2006; Butterfield 2007; Earman 2008; Wüthrich 2012; Callender and Wüthrich 2014).

But I shall confine attention only to the question whether the CSP harbors a genuine notion of Becoming. In fact, the CSP promises to support a dynamic conception of time in a growing block model of the universe, similar in basic principle to the one championed by Charlie Dunbar Broad (1923) or the one defended by Michael Tooley (1997). According to this conception, referred to in the literature under the rubric past-presentism or possibilism (Savitt 2014), the totality of the existent state of affairs depends on time and at each time all past and present objects, events, etc. exist but not those in the future. Analogously, according to the CSP, a causal set grows via a stochastic process of addition ("birth") of new elements, while the addition of a new element should not be regarded as taking place in time but rather as constituting time. This suggests the image of a universe in which the "sum total of existence is always increasing" - to quote Broad (1923, 66-67). In fact, Rafael Sorkin (2007, 157n), one of the leading proponents of the CSP, has made an explicit reference to Broad's view of time and John Earman has pointed out that the CSP "promises to transmute Becoming from a piece of speculative metaphysics to one of naturalized metaphysics" (Earman 2008, 159). On the other hand, Jeremy Butterfield has, in a passing remark, deflated these prospects (Butterfield 2007, 859).

By developing Butterfield's remark, which itself rests on a technical point well known to the proponents of the CSP, into a fully articulated argument, I shall argue that the CSP, with its classical stochastic process dynamics, does not substantiate past-presentism - at least, not at a higher degree than the familiar relativistic theories on continuous (smooth) spacetime manifolds. The proviso "with its classical stochastic process dynamics" is crucial here because there is as yet considerable uncertainty as to what a quantum dynamics for causal sets will look like.

The plan of the paper is as follows. Section 2 sketches the philosophical issue. Section 3 reviews the basics of the CSP - in particular, those elements that are relevant to the argument I shall offer. Section 4 expounds the central argument. And Section 5 wraps it up by stating more fully and more rigorously the conclusion.

2 As it is well known, the full geometric structure of a general relativistic spacetime cannot be recovered from its causal structure (viz. the structure induced by the order relation of connectibility of manifold points via timelike curves). The limitations for such a recovery were established by Malament (1977): roughly, the metrics of two general relativistic spacetimes that share the same causal structure may differ up to a conformal factor. (For a rigorous exposition, consult also Malament 2007, 270-271.) The CSP removes this "conformal ambiguity" by estimating the volume of a region in a general relativistic spacetime with the number of causal set elements "contained" in that region. The slogan is "geometry = order + number". For a more precise account, see Sorkin (2005). In this paper, philosophers will find explicitly stated philosophical motivations drawn from Riemann's views on physical geometry -views exploited later by Grünbaum $(1973,8-18)$ toward the thesis of the intrinsic metrical amorphousness of space and time continua- as well as references to Reichenbach and Robb. 


\section{World and Becoming}

According to a common intuition, time "passes" and the history of the universe gradually unfolds with the coming-to-be of new events. Modern physics, however, appears to be hostile toward this "intuitive metaphysics" of time. The familiar spacetime theories, relativistic or not, have models of the type $\left\langle M, G_{1}, \ldots, G_{n}\right\rangle$, where $M$ is a smooth 4-dimensional differentiable manifold and $G_{1}, \ldots, G_{n}$, for some natural number $n>0$, are geometric object fields specifying the geometry and the matter-energy content of spacetime. The points of the manifold $M$, as spacetime locations of idealized possible events, are assumed to be given, "once and for all", from the "beginning" in all four dimensions.

This mode of construction of physical theories about space and time sides, at first blush at least, with the metaphysical thesis that Williams ([1951] 1967) named "the doctrine of the manifold". Here is an excerpt from another paper by Williams in which the "doctrine" is proclaimed: ${ }^{3}$

What I advocate as 'the doctrine of the manifold', ... is simply a philosophical acceptance, as an ultimate literal truth about the way things are in themselves, of the conception that nature, all there is, was, or will be, 'is' (tenselessly) spread out in a four dimensional scheme of location relations which intrinsically are exactly the same, and hence in principle commensurate, in all directions, but which happen to be differentiated, in our neighborhood at least, by the de facto pattern of the things and events in them - by the lie of the land, so to speak. We are all perfectly familiar with the fact that the prodigious difference of the vertical dimension of space, with its terrifying asymmetry of up and down, above and below, from all those comparatively indifferent directions we call horizontal, is not due to any intrinsic difference between vertical and horizontal distances but only to a certain characteristic complex of matter and force in our vicinity whose 'grain', so to speak, runs one way and not the other. Just so, I argue, there is a somewhat more pervasive pattern of physical qualities and relations which constitutes the even more momentous oddity of the temporal direction, with its even more striking asymmetry of earlier and later, in contrast with all the socalled spatial directions (Williams 1965, 465).

The underlying philosophical debate is that between static and dynamic conceptions of the world and may be summarized thus. ${ }^{4}$ On a static conception of the world, what states of affairs exist does not depend upon time. Accordingly, change is not conceived as change in the totality of existent states of affairs over time; it is conceived, rather, in terms of the possession, by some object, or by the world as a whole, of different properties at different times. By contrast, according to a dynamic conception of the world, what states of affairs exist does depend upon time. Consequently, the totality of existing states of affairs a given object participates in may differ from one time to another. Such a difference

3 I owe the "discovery" of this particularly clear statement of the "doctrine of the manifold" to my reading of Savitt's (2002) - a paper devoted to unearthing a common ground between Williams's and Broad's later views.

4 Here I rely heavily on Tooley 1997, 13-16. 
is precisely what is conceived as change in the given object, as opposed to the mere possession by the object of different properties at different times. Similarly, change in the world as a whole is thought of, not in terms of the possession of different properties by different temporal slices of the world, but rather as a difference in the totality of states of affairs that exist as of different times.

Eternalists or block theorists ("Blockheads" in Earman's 2008 inspiring terminology) advocate a static conception of the world whereas past-presentists or growing block theorists (called "Broadheads" by Earman 2008) espouse a dynamic conception of the world. Eternalists, like Williams, believe that all past and future objects, events, etc. are as real as those of the present and that there neither were nor will be objects, events, etc. that do not exist now. By contrast, past-presentists, like Broad or Tooley, affirm the existence of all past or present objects, events, etc. but deny the existence of future objects, events, etc. ${ }^{5}$ Here is a telling excerpt from Broad's Scientific Thought:

When an event, which was present, becomes past, it does not change or lose any of the relations which it had before; it simply acquires in addition new relations which it could not have before, because the terms to which it now has these relations were then simply non-entities.

It will be observed that such a theory as this accepts the reality of the present and the past, but holds that the future is nothing at all. Nothing has happened to the present by becoming past except that fresh slices of existence have been added to the total history of the world. The past is thus as real as the present. On the other hand, the essence of a present event is, not that it precedes future events, but there is quite literally nothing to which it has the relation of precedence. The sum total of existence is always increasing, and it is this which gives the timeseries a sense as well as an order. A moment $t$ is later that a moment $t^{\prime}$ if the sum total of existence at $t$ includes the sum total of existence at $t^{\prime}$ together with something more (Broad 1923, 66-67).

Indeed, Broad distinguished three senses of the word 'change', with the third -the one he dubbed "absolute Becoming"- being the most fundamental:

I think that we must recognise that the word "change" is used in three distinct senses, of which the third is the most fundamental. These are (i) Change in the attributes of things, as where the signal lamp changes from red to green; (ii) Change in events with respect to pastness, as where a certain event ceases to be present and moves into the more and more remote past; and (iii) Change from future to present. I have already given an analysis of the first two kinds of change. It is clear that they both depend on the third kind (Ibid., 67).

As already noted, a similar view, but with significant differences, ${ }^{6}$ has been articulated recently by Tooley (1997). According to Tooley, the events in the world are connected via an asymmetric causal relation, with the causes producing their effects by "giving birth" to them, by "bringing them into existence". This

6 Mainly as regards the relative priority of tenseless over tensed concepts and facts and the crucial role of causation. 
conception of causation is combined with a substantivalist thesis according to which spacetime consists of points, thought of as contingent entities, with each spacetime point being the cause of birth of other spacetime points.

But I shall not dwell into more details of either Broad's or Tooley's views. I shall come directly to a well-known problem encountered by every attempt to implement a growing block model of the universe within classical general relativistic cosmology. ${ }^{7}$ It appears plausible to require that in a spacetime manifold, Becoming be represented by a family of spacelike hypersurfaces, indexed by the values of a global time function. But some models $\left\langle\mathrm{M}, g_{a b}, T_{a b}\right\rangle$ of classical general relativity do not admit a global time function $t: M \rightarrow \square, 8$ while those that do admit one admit an infinity of them. And one might make a case to deal with the first horn of the problem, i.e., the non-existence of a global time function in some general relativistic spacetimes. A necessary and sufficient condition for a spacetime of general relativity to admit a global time function is that it be stably causal. ${ }^{9}$ So one might confine attention to stably causal spacetimes and appeal to arguments that only such spacetimes are physically realizable. ${ }^{10}$ But the horn of the "embarrassment of riches" is thornier. Pastpresentism cannot tolerate such a "democracy". A global time function must be ontologically distinguished inasmuch as it demarcates "existence" from "nonexistence" by providing the temporary locus of "absolute Becoming".

As Earman has pointed out, the CSP suggests a promising way to deal with this problem (Earman 2008, 149). It offers a "mechanism" generating an increase in what Broad would regard as the "sum total of existence" and then examines whether this increase corresponds to the accretion of layers of "now" indexed by a global time function. So let me turn immediately to the basics of the CSP.

7 Earman presents this problem as a dilemma and canvasses the possible ways out, none of which appears trouble-free for the "Broadheads" (Earman 2008, 147-150). Earman's paper (2008) is the place to look for an admirably clear exploration of the prospects for a growing block model of the universe in both Newtonian and relativistic settings.

8 A global time function in a time-orientable spacetime of general relativity $\left\langle M, g_{a b}\right\rangle$ is a differentiable map $t: M \rightarrow \square$ whose gradient $\nabla^{a} t$ is a past-directed timelike vector field so that $t$ strictly increases along every future-directed timelike curve.

9 A time-orientable general relativistic spacetime $\left\langle M, g_{a b}\right\rangle$ is called stably causal if and only if there exists on $M$ a continuous, nonvanishing, timelike vector field $t^{a}$ such that the spacetime $\left\langle M, \tilde{g}_{a b}\right\rangle$ with $\tilde{g}_{a b}=g_{a b}-t_{a} t_{b}$ does not exhibit closed timelike curves. That a time-orientable general relativistic spacetime admits a global time function (as defined in the preceding Note) just in case it is stably causal is the content of Theorem 8.2.2 in Wald 1984, 198. See also Earman 1995, 166.

10 For example, Hawking and Ellis ([1973] 1989, 197) have argued that spacetimes that are not stably causal are not physically realizable along these lines: given that general relativity is expected to be the classical limit of a quantum theory of spacetime in which the metric does not have a definite "value" at each point, in order for a property of the spacetime to have physical significance it must be characterized by some "stability", i.e., must be as well a property of "nearby" spacetimes. Of course, such extrinsic justifications of causality conditions ultimately hinge upon debatable assumptions concerning putative "exotic possibilities" such as time travel. I shall not pursue this issue further. To start with, the reader may consult Earman 1995, ch. 6 and Arntzenius and Maudlin 2013. 


\section{Deep Structure of Spacetime: Causal Sets}

Discreteness and causal relatedness, the two pillars of the CSP, are reflected in the very definition of a causal set. A causal set or causet is a locally finite, partially ordered set ("poset"). Explicitly, a causal set is a structure $\langle c, \prec\rangle$, where $c$ is a set and $\prec$ is a binary relation on $c$ satisfying the following conditions: (i) for every $x, y, z \in c$, if $x \prec y$ and $y \prec z$, then $x \prec z$ (transitivity); (ii) for each $x \in c$, it is not the case that $x \prec x$ (irreflexivity); ${ }^{11}$ and (iii) for any $x, y, \in c,\{z \in c: x$ $\prec z \prec y\}$ is a finite set (local finiteness). Clearly, given a causet $\langle c, \prec\rangle$, the relation $\preceq$ defined by

For all $x, y \in c, x \preceq y$ if and only if $(x \prec y$ or $x=y)$

is a partial order on $c$. All the causal sets $\langle c, \prec\rangle$ we shall be considering below in the context of classical sequential growth satisfy the stronger than local finiteness property of being past finite: for every $x \in c$, the set $\{y \in c: y$ $\preceq x\}$ is finite. The statement ' $x \prec z$ ' is expressed by several suggestive locutions borrowed from mathematical, physical or genealogical terminology: ' $x$ precedes $y$ ', ' $y$ follows $x$, ' $x$ is in the past of $y$ ', $y$ is in the future of $x$, ' $x$ is an ancestor of $y$ ', ' $y$ is a descendant of $x$ ', etc.

The core idea of the CSP is that the elements of a causal set may be thought of as events in a discretized spacetime and the order relation as a causal relation. ${ }^{12}$ The basic assumption is that in the regime of very small scales (of the order of the Planck length $\left.l_{p}=\left(G \hbar / c^{3}\right)^{1 / 2}=10^{-33} \mathrm{~cm}\right)$ spacetime is no longer described by a semi-Riemannian metric on a smooth manifold, but by a causal set. To use an analogy suggested by Dowker (2005), just as ordinary matter appears to us "continuous" while in reality it consists of molecules and atoms, so spacetime appears to us "continuous" at large scales while in reality it is a causal set and the spacetime "continuum" of our experience is just an approximation to the underlying discrete ordered structure.

As an ordered set, every finite causal set can be represented graphically by a Hasse diagram. Draw a dot (vertex of a graph) to represent each element of the finite causal set and draw a line (edge of a graph) to represent each irreducible relation $x \prec y,{ }^{13}$ with the preceding element $x$ being represented by a dot drawn below the one representing the following element $y$. Figure 1 illustrates a very simple concrete example, with the elements of the causal set labeled by natural numbers.

11 The expression ' $x \nless y$ ' is often used to abbreviate 'It is not the case that $x<y$ '. Axiom (ii) for causal sets also goes by the name 'acyclicity'.

12 Philosophers should not read too much into the word 'causal' here. No worries should be triggered as to whether causation is indeed a transitive relation (see, e.g., Hitchcock 2001) or as to whether the intended use of the term captures a single robust notion of causation familiar from the philosophical tradition, if indeed there is one (see, e.g., Psillos 2007). One should have, rather, in mind the concept of causal relatedness or causal connectibility as it is employed in relativistic spacetime physics.

13 The relation between two causet elements is said to be irreducible -in the jargon, a link-if and only if it is not implied by other such relations via transitivity. 


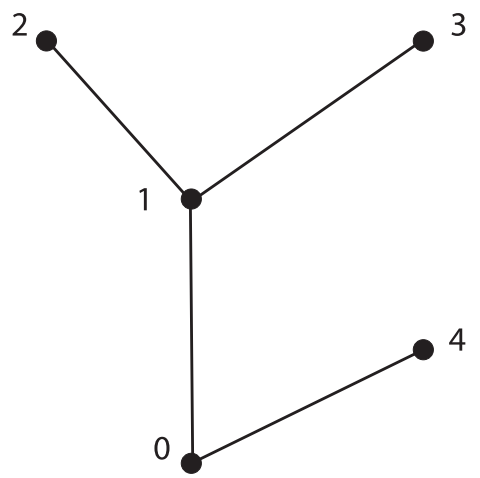

Figure 1. Hasse diagram of a labeled finite causal set

If you have trouble imagining the "spacetime continuum of our experience" to "arise" from a simple discrete structure like the one depicted in Figure 1, take into consideration that, according to rough estimates (Dowker 2006, 5), in the causal set underlying $1 \mathrm{~cm}^{3} \times 1 \mathrm{~s}$ of the spacetime continuum you should expect some $10^{143}$ elements. (Not that such an estimate can really assist the imagination!)

Leaving aside flights of the imagination, I turn immediately to the tedious chore of reviewing a few basic definitions needed in the rest of the paper. Let $c$ be a causal set. The past of an element $x \in c$ is the set of all elements that precede it in $c$, past ${ }_{c}(x)=\{y \in c: y \prec x\}$. And an element $x \in c$ is called maximal if and only if it is to the past of no element - i.e., $x \notin$ past $_{c}(y)$ for every $y \in c$. Let, now, $a$ be a subset of $c, a \subseteq c$. The past of the subset $a$ of $c$ is just the union of the pasts of its elements, past $c_{c}(a)=\cup_{x \in a}$ past $_{c}(x)$. Of course, $a$ is said to be a chain just in case it is linearly ordered - i.e., any two distinct elements of $a$ are related by the relation $\prec$ of precedence $(x \prec y$ or $y \prec x$ for any $x, y \in a$ with $x \neq y)$. On the other hand, $a$ is called a stem if and only if it is finite and contains its own past (past ${ }_{c}$ $(a) \subseteq a) \cdot{ }^{14}$

Now, as I have mentioned already, the exact final formulation of a quantum dynamics for causal sets is still an open question. But as a first step in the direction of formulating such a dynamics, Sorkin and collaborators have worked out a dynamics of a classical stochastic process in discrete stages, a classical sequential growth (CSG) dynamics. ${ }^{15}$ The basic idea is this. Starting from the empty set, a causal set grows via a stochastic process at each stage of which a new element is added to the already existing causal set, with the process running to infinity. Accordingly, the elements of any causal set, be it finite or infinite, may be labeled by natural numbers. Specifically, a natural labeling of a causal set $c$

14 To illustrate, if $c$ is the simple 5-element causet depicted in Figure 1 , then past $(0)=\varnothing$, past (2) $\{0,1\}$, past p $_{c}(4)=\{0\}$, past $(\{1,3\})=\{0,1\}$, past $c_{c}(\{0,1\})=\{0\}$, and so on. The elements 2 , 3,4 are the maximal elements of $c$. The subset $\{0,1,2\}$ is a chain whereas $\{2,4\}$ is not. And the subsets $\{0,1,2\},\{0,1,3\},\{0,4\}$, and $\{0,1\}$ are stems whereas $\{1,2\},\{0,3\}$, and $\{4\}$ are not. Note also that Rideout and Sorkin $(1999,2)$ use the term 'partial stem' for what is here called simply a stem.

15 The dynamics I sketch in what follows is the one formulated by Rideout and Sorkin 1999. 
is a bijective mapping of the initial segment of the set of natural numbers $\square=$ $\{0,1,2, \ldots\}$ whose cardinality equals that of $c$-hence, of $\square$ itself, if $c$ is infiniteonto $c$ that preserves order. That is, for a causal set $c$ with $n$ elements, a natural labeling is just a $1-1$ and onto map

$$
l:\{0,1, \ldots, n-1\} \rightarrow c
$$

such that for all $x, y \in c$, if $x \prec y$, then $l(x)<l(y)$. By convention, a natural labeling of a causet expresses an order of birth we attribute to its elements, but carries no intrinsic physical significance (see below). ${ }^{16}$

Obviously, a causal set may admit more than one labelings. A labeled causal set $\tilde{c}=\left\langle c, l_{\tilde{c}}\right\rangle$ is just a causal set, $c$, together with one of its labelings, $l_{\tilde{c}} .{ }^{17}$ Given two labeled causal sets, $\tilde{c}=\left\langle c, l_{\hat{c}}\right\rangle$ and $\tilde{b}=\left\langle b, l_{\tilde{b}}\right\rangle$, of the same cardinality, say $k^{18}$ we shall say that $\tilde{c}$ and $\tilde{b}$ are $k$ - label variants and we shall write $\tilde{c} \square_{k} \tilde{b}$ if and only if $l_{\tilde{b}} \circ l_{\tilde{c}}^{-1}: c \rightarrow b$ is an isomorphism with respect to the order relations in the causal sets $c$ and $b$. In such a case, $\tilde{c}$ and $\tilde{b}$ are isomorphic ordered structures differing only in their labelings. Evidently, for each $k=0,1,2, \ldots, \infty,{ }^{19}$ the binary relation $\mathrm{Q}_{k}$ of $k$ - label variance is an equivalence relation on the set of labeled causal sets with cardinality $k$.

The dynamical law in a CSG setting for causal sets reduces to an assignation of probability to each transition from every finite causal set to each causal set that can be formed from it by the addition of exactly one element. The RideoutSorkin (RS) models of such a dynamics are required to satisfy four postulates I shall briefly present next.

First is the requirement of internal temporality: each element of a causal set is born either to the future of, or unrelated to, all existing elements; no element can arise to the past of an existing element. Rideout and Sorkin phrase the rationale thus:

The phenomenological passage of time is taken to be a manifestation of this continuing growth of the causet. Thus we do not think of the process as happening "in time," but rather as "constituting time" ... (Rideout and Sorkin 1999, 2).

Put another way, physical time is defined by the intrinsic ordering of a causal set.

The second postulate is the requirement of discrete general covariance: the net probability of formation of any particular finite causal set is independent of the order of birth we attribute to its elements (i.e., of each natural labeling of its

16 Henceforth, I shall consider only natural (i.e., order-preserving) labelings of causets but I shall often drop, for brevity, the qualification 'natural'.

17 A note on notation. I use small case letters from the beginning of the Latin alphabet $(a, b$, $c, \ldots)$, sometimes embellished with accents, to denote causets. For each $n \in \mathbb{Q}, \Omega(n)$ is the set of all causets with $n$ elements. $\Omega(\square)=\cup_{n \in \square} \Omega(n)$ is the set of all finite causets. And $\Omega$ is the set of all infinite "completed" causets, i.e., those that result "when" the sequential process of accretion of new elements "runs to completion". A tilde above the symbols for individual causets $(\tilde{a}, \tilde{b}, \tilde{c}, \ldots)$ and above the symbols for sets of causets $(\tilde{\Omega}(n), \tilde{\Omega}(\square), \tilde{\Omega})$ signifies natural labeling.

18 Clearly, by the cardinality of a labeled causet $\tilde{c}=\left\langle c, l_{\tilde{c}}\right\rangle$ I just mean the cardinality of $c$.

19 Mathematicians will forgive this notation and, in particular, my opting for ' $\infty$ ' instead of ' $\mathrm{N}$.' 
elements). Consider the set $\Omega(\square)$ of all finite causal sets, partially ordered by the relation $\triangleleft$, where $b \triangleleft c$ just in case $c$ can grow out of $b$ via CSG. ${ }^{20}$ And visualize the growth of a causal set in terms of directed paths in the graph of $\langle\Omega(\square), \triangleleft\rangle$ (a Hasse diagram of Hasse diagrams). Then discrete general covariance entails that any two paths with the same initial and final end points have the same product of transition probabilities. Figure 2 illustrates this in a simple example. ${ }^{21}$

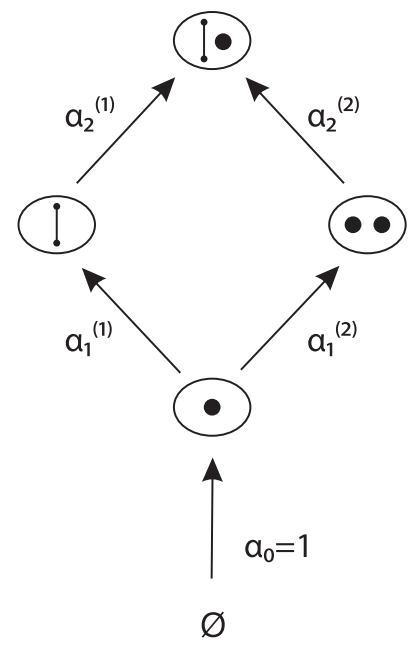

Figure 2. Discrete general covariance in CSG

CSG starts with the transition from the empty set to the 1-element causet - a transition assigned the probability value $\alpha_{0}=1$ because "the universe does exist". Thereafter there are two paths, indicated by the superscripts '(1)' and '(2)', that can lead to the formation of the 3-element causet depicted at the top, each involving the intermediate formation of a different 2-element causet. Discrete general covariance demands that the products of transition probabilities along these two paths be equal: $\alpha_{1}{ }^{(1)} \alpha_{2}{ }^{(1)}=\alpha_{1}{ }^{(2)} \alpha_{2}{ }^{(2)}$.

Thus a labeling of a causal set, even if natural, carries no physical significance. It stands for a kind of "external" or "extrinsic" time and introduces a gauge element into the theory because we use it to describe the dynamics of the growth of causal sets. The condition of discrete general covariance expresses what would here correspond to "invariance under gauge transformations". Indeed, the counterpart of a natural labeling of a causal set in a spacetime continuum would be a coordinate system $\left(x^{i}\right)_{i=0,1,2,3}$ whose first coordinate $x^{0}$ would be everywhere timelike so that it could serve to foliate spacetime into a family of spacelike hypersurfaces. ${ }^{22}$ And, of course, physics is independent of the choice of coordinates.

20 More precisely, for any $b, c \in \Omega(\square), b \triangleleft c$ if and only for some $n \in \square$ there exist $a_{0}, a_{1}, \ldots$, $a_{n} \in \Omega(\square)$ such that $a_{0}$ is isomorphic to $b, a_{n}$ is isomorphic to $c$, and for every $i \in\{0,1, \ldots$, $n\}, a_{i+1}$ can be formed by accreting a single element to $a_{i}\left(a_{i+1}\right.$ is a "child" of $a_{i}$, to adopt the genealogical vocabulary dear to the proponents of the CSP).

21 It is a mere reproduction of Figure 3 of Earman's $(2008,157)$.

22 See also Rideout and Sorkin 1999, 2 n3. 
The third requirement imposed on the RS models for CSG dynamics implements the classical idea that events occurring in some "part" of a causal set are influenced only by that "portion" of the causal set lying to their past. It is the condition of Bell causality: the probability of a particular addition of a new element to a causal set depends only on the past of the new element and is not affected by elements in spacelike separation. The mathematical formulation provided by Rideout and Sorkin looks a bit convoluted at first blush (Rideout and Sorkin 1999, 6-7). It starts with a definition: for every $n \in \square$, the precursor set of the transition from $b \in \Omega(n)$ to $c \in \Omega(n+1)$ induced by the birth of a new element $x$ is defined by

$$
\text { precursor }(b \rightarrow c)=\operatorname{past}_{c}(x) \subseteq b .
$$

Bell causality demands that if for each $j \in\{1,2\}, c \rightarrow c_{j}$ is a transition from $c$ $\in \Omega(n)$ to $c_{j} \in \Omega(n+1)$, then

$$
\frac{\operatorname{Pr}\left(c \rightarrow c_{1}\right)}{\operatorname{Pr}\left(c \rightarrow c_{2}\right)}=\frac{\operatorname{Pr}\left(b \rightarrow b_{1}\right)}{\operatorname{Pr}\left(b \rightarrow b_{2}\right)},
$$

where $b=\operatorname{precursor}\left(c \rightarrow c_{1}\right) \cup \operatorname{precursor}\left(c \rightarrow c_{2}\right)(b \in \Omega(m)$ for some $m$ $\leq n)$ and $b_{j}$ is the causal set resulting from $b$ via the addition of a new element in the same manner as in the transition $c \rightarrow c_{j}\left(b_{j} \in \Omega(m+1)\right)$. Figure 3 offers a simple illustration: the diagram on the left depicts two possible transitions from a 3 -element causet $c$ to two different 4 -element causets, $c_{1}$ and $c_{2}$, while the diagram on the right depicts the causets $b, b_{1}, b_{2}$ associated with these transitions as in the aforementioned stipulations.
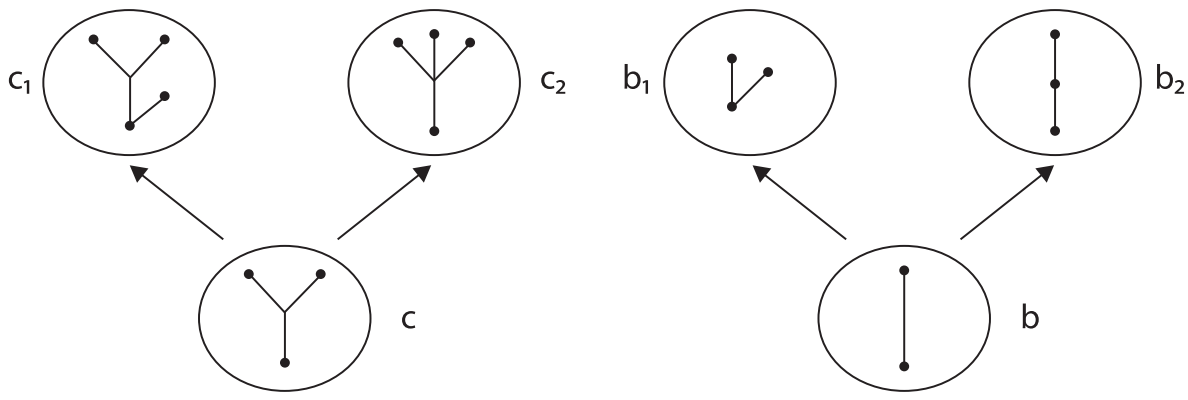

Figure 3. Bell causality in CSG

In this example, of course, the satisfaction of Bell causality amounts again to the satisfaction of relation (4).

The last requirement assumed to be satisfied by the RS models ensures that a CSG dynamics for causal sets be a Markov process. ${ }^{23}$ It is the Markov sum rule: the sum of all transition probabilities issuing from a given causal set must be equal to 1 .

23 It might be helpful to recall here the slogan characterizing Markov processes: in a Markov process, the future and the past are independent if one conditionalizes on the present. 
It has been shown that these four conditions are sufficiently restrictive to determine every transition probability from a causal set with $n$ elements to a causal set with $n+1$ elements, for any $n \in \square$, leaving just one free parameter for each stage of the stochastic process. Under the assumption that no transition probability vanishes, ${ }^{24}$ one can derive that an arbitrary transition probability $\alpha_{n}$ from a member of $\Omega(n)$ to a member of $\Omega(n+1)$ may be computed by employing the formula

$$
\alpha_{n}=\frac{\sum_{j=m}^{\varpi}\left(\begin{array}{c}
\varpi-m \\
\varpi-j
\end{array}\right) t_{j}}{\sum_{k=0}^{n}\left(\begin{array}{l}
n \\
k
\end{array}\right) t_{k}},
$$

where $\varpi$ stands for the cardinality of the precursor set of the transition, $m$ is the number of maximal elements in the precursor set of the transition, and $t_{i}(i$ $=0,1,2, \ldots)$ are free parameters satisfying $t_{0}=1$ and $t_{i} \geqslant 0$.

Needless to say, all of the above provide no more than a mere sketch of a central theme developed within the CSP. But I shall forego the discussion of other technical aspects of the CSP and turn to the exploration of its relation to growing block views of the universe.

\section{General Covariance: Block Universe}

Again, recall what the promise is: the CSP will provide a physical mechanism implementing a genuine notion of Becoming. Here is, in more detail, Earman's forceful way of putting the matter:

Apart from the hoary philosophical debates about the reality of the future, the growing block model is given new life by the causal set approach to quantum gravity. By providing a physical mechanism for producing growth and Becoming, this approach promises to transmute Becoming from a piece of speculative metaphysics to one of naturalized metaphysics. Furthermore, the causal set approach avoids any appearance of parasitism on block models, since it makes classical relativistic spacetime an emergent feature of Becoming, something Broad would have no doubt applauded (Earman 2007, 159-160).

And, certainly, this assessment agrees not only with the imagery residing at the core of the CSP but also with declarations made by some of its proponents. I shall now argue that the enthusiasm all this may instigate in the hearts of growing block fans should be tempered, once all the consequences of the principle of discrete general covariance are unraveled.

In the context of classical general relativity on differentiable manifolds, the requirement of general covariance enforces diffeomorphism-invariance on

24 Rideout and Sorkin (1999), whose approach and results I present here, imposed this assumption but Varadarajan and Rideout (2006) removed it. Of course, once the assumption of nonzero transition probabilities is removed, some of the physical postulates (notably, Bell causality) must be reformulated to make mathematical sense. 
the dynamical models and coordinate-independence on the observables. We have mentioned already the way in which discrete general covariance restricts the stochastic dynamics of causal sets. What can we say about observables? Alternatively, what sort of questions is the available causal set theory expected to give answers to and how?

For comparison, let us start from a more familiar probabilistic physical theory - namely, quantum mechanics. The elementary questions quantum mechanics can give answers to have the form: 'Will a measurement of the observable $O$ conducted on a quantum system in the state $|\Psi\rangle$ yield a result in the (measurable) set $\Delta$ of real numbers?' And the answers are probabilistic: 'Yes, with probability $\operatorname{Pr}_{|\Psi\rangle}(O, \Delta)$, with the probability value being computed courtesy of some variant of Born's statistical algorithm. But the causal set theory under discussion here appears to be a physical theory of a quite different ilk. ${ }^{25}$ Its "empirical claims" -i.e., the claims associating (some of) its models with the world- are intended to pertain to the universe as a whole and the dynamics is supposed to describe possible entire histories of the universe (possible universes). Accordingly, the relevant elementary questions inquire as to whether a given causal set $c$ belongs to this or that possible history. Such questions assume the general form: 'Will the CSG of the causal set $c$ "end up" forming an infinite ("completed") causal set that satisfies the predicate $\Phi$ ?' where ' $\Phi$ ' stands for some predicate of interest. For example, the symbolic statement ' $\Phi(a)$ ' may stand for ' $a$ contains a 4-chain' or ' $a$ contains an element whose ancestors and descendants jointly exhaust the remainder of $a, 26$ etc. And the answers are, again, of probabilistic nature: 'Yes, with probability $\mu(A)$ ', where $A$ is that subset of $\Omega$ whose members satisfy the appropriate logical combination of predicates at hand.

However, we have not as yet shown how the probability measure $\mu$ is constructed. In essence, we have not as yet defined mathematically the relevant stochastic process. Indeed, the family $\left\{\alpha_{n}: n \in \square\right\}$ of transition probabilities for finite causal sets, given by (5) above, does not by itself constitute a stochastic process. Recall that, in general, a stochastic process is a family $\left\{X_{t}: t \in T\right\}$ of random variables on a probability space $\langle\Omega, R, \mu\rangle$, indexed by (continuous or discrete) "time" $t{ }^{27}$ Furthermore, for the measure $\mu$ to be defined via Kolmogorov's theorem, all the finite-dimensional distributions $\mu_{t_{1} \ldots t_{k}},\left\langle t_{1}, \ldots, t_{k}\right\rangle \in T, k=1,2, \ldots$, are needed. ${ }^{28}$

25 The differences are mitigated if quantum mechanics is construed along the lines of a suitable version of the consistent histories interpretation, favored, of course, by adherents to the CSP.

26 In the jargon, such an element is called a post. From the cosmological viewpoint, whether the universe's causal set will develop a post amounts to whether the universe will recollapse.

$27 \Omega$ is the sample space, $\mathrm{R}$ is a $\sigma$-algebra $(\sigma$-field) in $\Omega$, and $\mu: \mathrm{R} \rightarrow \square$ is a probability measure on R. I shall not rehearse here the well-known definitions of these concepts or of the concept of a random variable.

28 If $\left\{X_{t}: t \in T\right\}$ is a stochastic process in a probability space $\langle\Omega, R, \mu\rangle$, then for each $k$-tuple $\left\langle t_{1}\right.$, ..., $\left.t_{k}\right\rangle$ of distinct elements of $T$, the random vector $\left\langle X_{t_{1}}, \ldots, X_{t_{k}}\right\rangle$ has, over $\square^{k}$, some distribution $\mu_{t_{1} \ldots t_{k}}: \mathfrak{R}^{k} \rightarrow \square: H \mapsto \mu\left(\left\{\omega \in \Omega:\left\langle\mathrm{X}_{t_{1}}(\omega), \ldots, X_{t_{k}}(\omega)\right\rangle \in H\right\}\right)$, where $\mathfrak{R}^{k}$ is the $\sigma$-algebra of $k$-dimensional Borel sets $\left(\Re=\mathfrak{R}^{1}\right)$. The probability measures $\mu_{t_{1} \ldots t_{k}}$ are the finite-dimensional distributions of the stochastic process. Kolmogorov's existence theorem says, conversely, 
It follows that in order to define with mathematical consistency the stochastic process representing CSG for causal sets, one should envision the birth of new elements to run ad infinitum as is, indeed, the case in RS universes. And this amounts to a first move of retreat vis-à-vis the block-universe idea as Sorkin himself readily concedes:

In order to define [the measure] consistently, one must take [the sample space] to be a space of infinite causets, ones for which the growth process has "run to completion". We meet here with an echo of the block-universe idea, that is in effect built into mathematicians' formalisation of the concept of stochastic process (Sorkin 2007, 160n8).

Be this as it may, the proponents of the CSP have done all the necessary mathematical work. ${ }^{29}$

In short, the first step toward the construction of the relevant probability space has as follows. Take $T=\square$ as the index set for "external" or "extrinsic" "time". Let the sample space be the set $\tilde{\Omega}$ of labeled infinite ("completed") causal sets. For each labeled finite causal set $\tilde{b}$ of cardinality $\operatorname{card}(b)=n \in \square$, the cylinder set with base $\tilde{b}$ (of rank $n$ ) is just the set of all labeled infinite causal sets whose first $n$ elements (those labeled $0,1, \ldots, n-1$ ) form a causal set isomorphic to $\tilde{b}$ (with the same labeling) - symbolically:

$$
\begin{aligned}
\operatorname{cyl}(\tilde{b})= & \{\tilde{c} \in \tilde{\Omega}: \text { the first card }(b) \text { elements of } \tilde{c} \text { form an } \\
& \text { isomorphic copy of } \tilde{b} \text { with the same labeling }\} .
\end{aligned}
$$

Accordingly, the $\sigma$-algebra $\tilde{R}$ in $\tilde{\Omega}$ is defined as the $\sigma$-algebra generated by all the cylinder sets $\operatorname{cyl}(\tilde{b}), \tilde{b} \in \Omega(\square)$, i.e., as the smallest $\sigma$-algebra of subsets of $\tilde{\Omega}$ containing all these cylinder sets. It remains to define the appropriate probability measure on $\tilde{R}$. The transition probabilities $\alpha_{n}, n \in \square$, given by (5) above, determine the probability of formation of each labeled finite causal set (equal to the product of the transition probabilities corresponding to the individual births described by the labeling) and, consequently, a real function $\tilde{\mu}$ on the set of all cylinder sets. This function can, then, be extended to a probability measure $\tilde{\mu}$ on the entire $\sigma$-algebra $\tilde{R}$ via the standard mathematical procedures

that if a given system of such measures satisfies two consistency conditions, then there exists a stochastic process having these finite dimensional distributions. More precisely, if $T$ is an arbitrary set and to each $k=1,2, \ldots$ and each $\left\langle t_{1}, \ldots, t_{k}\right\rangle \in T^{k}$ there corresponds a joint distribution function $\mu_{t_{1} \ldots t_{k}}: \Re^{k} \rightarrow \square$ so that all the $\mu_{t_{1} \ldots t_{k}}$ satisfy the following two conditions:

(a) For all $H_{1}, \ldots, H_{k} \in \Re$ and every permutation $\pi$ of $\langle 1,2, \ldots, k\rangle$,

$$
\mu_{t_{1} \ldots t_{k}}\left(H_{1} \times \ldots \times H_{k}\right)=\mu_{t_{\pi(1)} \ldots t_{\pi(k)}}\left(H_{\pi(1)} \times \ldots \times H_{\pi(k)}\right) \text {, and }
$$

(b) For all $H_{1}, \ldots, H_{k} \in \mathfrak{R}$,

$$
\mu_{t_{1} \ldots t_{k-1}}\left(H_{1} \times \ldots \times H_{k-1}\right)=\mu_{t_{1} \ldots t_{k-1} t_{k}}\left(H_{1} \times \ldots \times H_{k-1 x} \times H_{k}\right) \text {, }
$$

then there exists on some probability space $\langle\Omega, R, \mu\rangle$ a stochastic process $\left\{X_{t}: t \in T\right\}$ having the $\mu_{t_{1} \ldots t_{k}}$ as its finite-dimensional distributions. Billingsley (1995, ch. 7, sec. 36) is a standard reference for all these mathematical niceties.

See Brightwell et al. 2002; 2003; as well as Dowker and Surya 2006. 
underpinning Kolmogorov's theorem. Thus is obtained the probability space $\langle\tilde{\Omega}$, $\tilde{\mathrm{R}}, \tilde{\mu}\rangle$ pertaining to the labeled infinite ("completed") causal sets. ${ }^{30}$

Still, this approach is not "covariant". ${ }^{31}$ The probability space $\langle\tilde{\Omega}, \tilde{\mathrm{R}}, \tilde{\mu}\rangle$ affords probabilistic answers to questions that are not covariant inasmuch as they implicate, for admitting a definite probabilistic answer, some particular labeling. ${ }^{32}$ Yet, the postulate of discrete general covariance requires independence from labeling. The elements of a causal set are not intrinsically individuated and, consequently, for each labeled causal set the only representation endowed with physical significance is the isomorphism equivalence class it belongs to.

The switch to a covariant probability space is brought about in the following manner. Take the set $\Omega$ of unlabeled infinite ("completed") causal sets for sample space. Consider the relation $\square_{\infty}$ of $\infty$-label variance in $\tilde{\Omega}$ discussed in the previous Section and deem a subset $A$ of $\tilde{\Omega}$ to be covariant just in case along with each of its members, $A$ contains all members of $\tilde{\Omega}$ equivalent (isomorphic) to it: for all $\tilde{c}, \tilde{c}^{\prime} \in \tilde{\Omega}$, if $\tilde{c} \in A$ and $\tilde{c} \square \tilde{c}^{\prime}$, then $\tilde{c}^{\prime} \in A$. Let $\mathrm{R}$ be the collection of all subsets $A$ of $\tilde{\Omega}$ that are both $\tilde{\mu}$-measurable $(A \in \tilde{R})$ and covariant in the aforementioned sense. Note that each $A \in \mathrm{R}$, although "originally" a subset of $\tilde{\Omega}$, it may also be regarded as a subset of $\Omega$ because it is relabeling invariant. Further, it is not hard to prove that R constitutes a $\sigma$-algebra of subsets of $\tilde{\Omega}$ (and of $\Omega$ ) and, indeed, a sub- $\sigma$-algebra of $\tilde{R}$ since clearly $R \subseteq \tilde{R}$. Therefore, the restriction of the measure $\tilde{\mu}$ to $\mathrm{R}$ yields a measure $\mu=\tilde{\mu} \mid \mathrm{R}$ on the measurable space $\langle\Omega, \mathrm{R}\rangle$ for unlabeled infinite ("completed") causal sets (i.e., $\mu(A)=\tilde{\mu}(A)$ for every $A \in \mathrm{R}$ ). Thus we get the covariant probability space $\langle\Omega, R, \mu\rangle$ for the RS models of CSG "covariant" in the sense that the members of R correspond exactly to the physical meaningful, according to discrete general covariance, questions the dynamics provides probabilistic answers to by means of $\mu .{ }^{33}$

However, the physical significance of the members of the covariant $\sigma$-algebra $\mathrm{R}$ is not clear. The question or predicate an arbitrary $A \in \mathrm{R}$ corresponds to cannot,

30 The relevant stochastic process involves the family $\left\{X_{n}: n \in \square\right\}$-recall $T=\square-$ of random variables that are defined in this fashion. Identify each $\tilde{c} \in \tilde{\Omega}$ with the sequence $\left\langle\tilde{c}_{0}, \tilde{c}_{1}, \tilde{c}_{2}, \ldots\right.$, $\left.\tilde{c}_{n}, \ldots\right\rangle, \tilde{c}_{n} \in \tilde{\Omega}(n), n \in \square$, representing the successive stages of its formation out of the empty set according to its labeling ( $\tilde{c}_{0}=\varnothing$ and $\tilde{c}_{1}$ is the singleton comprising the element labeled 0$)$. Then, for each $n \in \square$, set $X_{n}: \Omega \rightarrow \tilde{\Omega}(\square): \tilde{c} \mapsto \tilde{c}_{n}$.

31 Henceforth, I shall unashamedly apply the predicate 'covariant' to questions, $\sigma$-algebras, probability spaces, etc., hoping that what is meant in each case becomes manifest from what I write in the context.

32 'Does $\tilde{c}$ comprise a chain before the $13^{\text {th }}$ stage of its growth?' is an example of such a question.

33 Alternatively, we could construct the quotient space $\langle\tilde{\Omega} / \square, \tilde{R} / \square, \tilde{\mu} / \square\rangle$ of the probability space $\langle\tilde{\Omega}, \tilde{R}, \tilde{\mu}\rangle$ with respect to the equivalence relation $\square$. (Here I write ' $\square$ ' instead of ' $\square$ ', to reduce clutter in the notation.) For each $\tilde{c} \in \tilde{\Omega}$, let $\tilde{c} / \square$ be the equivalence class of $\tilde{c}$ with respect to $\square$, i.e., the set of all $\tilde{b} \in \tilde{\Omega}$ such that $\tilde{b} \square \tilde{c}$. Identify $\tilde{\Omega} / \square$ with the set $\{\tilde{c} / \square: \tilde{c} \in \tilde{\Omega}\}$ and consider the function $p: \tilde{\Omega} \rightarrow \tilde{\Omega} / \square: \tilde{c} \mapsto p(c)=\tilde{c} / \square$. Define $\tilde{R} / \square$ by stipulating that for every $A \subseteq \tilde{\Omega} / \square, A \in \tilde{\mathrm{R}} / \square$ if and only if $p^{-1}[A] \in \tilde{\mathrm{R}}$, where $p^{-1}[A]=\{\tilde{c} \in \tilde{\Omega}: p(\tilde{c}) \in A\}$. Lastly, define the measure $\tilde{\mu} / \square$ on the quotient measurable space $\langle\tilde{\Omega} / \square, \tilde{R} / \square\rangle$ by demanding that $(\tilde{\mu} / \square)(A)=\tilde{\mu}\left(p^{-1}[A]\right)$ for each $A \in \tilde{R} / \square$. As expected, $\langle\Omega, R, \mu\rangle$ can be naturally identified with $\langle\tilde{\Omega} / \square, \mathrm{R} / \square, \tilde{\mu} / \square\rangle$ via the identification of each member $c$ of $\Omega$ with the member $\tilde{c} / \square$ of $\tilde{\Omega} / \square$ where $\tilde{c}=\left\langle c, l_{\tilde{c}}\right\rangle$ with $l_{\tilde{c}}$ some labeling of $c$. 
in general, be couched in terms familiar to the available theory describing causal set structure and growth. Yet, some members of R do have an apparent physical significance. These are the so-called "stem sets" defined by arbitrary unlabeled finite causal sets. For any $b \in \Omega(\square)$, define the stem set of $b$ by

$$
\operatorname{stem}(b)=\{c \in \Omega: c \text { contains a stem isomorphic to } b\}
$$

That is, stem $(b)$ comprises exactly those unlabeled infinite ("completed") causal sets for each of which there exists a natural labeling such that the first $\operatorname{card}(b)$ elements form a causal set isomorphic to $b$. That stem $(b) \in \mathrm{R}$, for every $b \in \Omega(\square)$, follows from the fact that $\operatorname{stem}(b)$ (viewed as a subset of $\tilde{\Omega}$ ) is a countable union of cylinder sets:

$$
\operatorname{stem}(b)=U\{\operatorname{cyl}(\tilde{c}): \tilde{c} \in \tilde{\Omega}(\square) \text { and } b \text { is a stem in } \tilde{c}\} .
$$

Clearly, for each $b \in \Omega(\square)$, the associated member stem $(b)$ of $\mathrm{R}$ corresponds to a question whose physical meaning is evident: for every $c \in \Omega$, the question ' $c \in \operatorname{stem}(b)$ ?' just means 'Does $c$ contain a stem isomorphic to $b$ ?' And the answer afforded by the theory is, of course, probabilistic: 'Yes, with probability $\mu(\operatorname{stem}(b))$.

But are all infinite ("completed") causal sets characterized by their stems? The exact mathematical answer is "Almost, yes!" Here is how this answer is substantiated. Call an infinite ("completed") causal set a "rogue" if and only if there exists another infinite ("completed") causal set that is not isomorphic to the former although it shares with it exactly the same stems: $c \in \Omega$ is a rogue if and only if there exists a $c^{\prime} \in \Omega$ such that $c^{\prime}$ is not isomorphic to $c$ but for every $b \in \Omega(\square), c \in \operatorname{stem}(b)$ just in case $c^{\prime} \in \operatorname{stem}(b)$. Put the other way around, every infinite ("completed") causal set that is not a rogue is characterized up to isomorphism by its stems. Now, a remarkable theorem demonstrated by Brightwell et al. (2003, 4, Proposition 1) affirms that in any CSG dynamics, the set $\Theta$ of all rogues in $\Omega$ has measure zero, $\mu(\Theta)=0$. Therefore almost every infinite ("completed") causal set produced by a CSG dynamics is characterized up to isomorphism by its stems.

The upshot of all this can be stated thus. With the exception of a set of infinite ("completed") causets of measure zero, all that can be meaningfully said about an infinite ("completed") causet may, once appropriately analyzed, be expressed by reference to its stems. In this sense, the stem questions of the form 'c $\in \operatorname{stem}(b)$ ?', with $c \in \Omega$ and $b \in \Omega(\square)$, "virtually" exhaust the set of covariant and physically meaningful elementary questions a causal set cosmology built on CSG is expected to tackle.

I shall now argue that precisely this aspect of CSG prohibits the causal set cosmology coupled to it from buttressing a robust notion of Becoming consonant with a growing block model of the universe. My argument rests on the following philosophical presuppositions. ${ }^{34}$ A physical theory hosts a genuine notion of

34 The argument is tailored after the so-called "truthmaker" or "grounding" objection(s) against presentism in the philosophy of time. Cf. Crisp 2003, 236-242; Rea 2003, 261-268. 
Becoming, consonant with a growing block model of the universe, only if it distinguishes "stages of Becoming" so that at each such stage the entire history of the world is divided into a part that "has already become" or "is already definite" and a part that "has not as yet become" but is (temporarily) "indefinite". And if truth supervenes on being, as David Lewis (2001) has suggested, such a theory should accord physical significance to propositions whose truth-values supervene on states of affairs that have already become as of some such stage. Thus we arrive at the following necessary condition, $\mathrm{I}$ dub $[\mathrm{N}]$ for future reference.

\section{CONDITION [N]. A physical theory T supports a notion of Becoming consonant with a growing block model of the universe only if $T$ posits a family $\left\{t_{i}: i \in I\right\}$ of stages and assigns physical significance to at least one proposition $p$ such that for some stage $t_{i}$ the following holds: for all possible according to Tworlds $w$ and $w^{\prime}$, if $p$ is true in $w$ but false in $w^{\prime}$, then $w$ and $w^{\prime}$ disagree with respect to facts up to $t_{i}$.}

The question we have to address now concerns the way in which "stages of Becoming" may be represented in a CSG dynamics. Of course, as causal sets are intended to portray discrete spacetimes, these stages should admit $\square$ as index set. But what sort of structure in a growing causal set is going to represent the temporary locus of Becoming at the $n^{\text {th }}$ stage for $n \in \square$ ? There appear only two choices. According to the first, call it "Choice 1", this structure may include (for sufficiently large $n$ ) more than one causet elements, none of which is an ancestor or descendant of another. According to the second, "Choice 2", the temporary locus of Becoming at each stage contains exactly one causet element. Intuitively, Choice 1 is intended to salvage, in a discrete spacetime, a surrogate for "hupersurface Becoming" in continuous spacetimes, whereas Choice 2 allies with the idea of "localizing" Becoming and the present. Neither choice supports the thesis that the CSP harbors a notion of Becoming that is both genuine by the standards of growing block theorists and novel in the sense that it has not been put forth in the context of relativistic theories of spacetime continua. Or so I shall argue.

Take up Choice 1 first. The most plausible way to spell it out mathematically seems to be this. Consider any infinite ("completed") causet $c$. Define the level of an element $x$ in $c$ as the maximum length of a chain in $c$ with top element $x .^{35}$ Given that causets are past finite, every element in $c$ has some finite level. For each $n \in \square$, define the $n^{\text {th }}$ level of $c$ as the set of all elements of $c$ of level $n$ and recognize it as a single stage of Becoming in the CSG producing $c .{ }^{36}$ Along this line, the part of $c$ that has already become as of a given stage comprises exactly those elements of $c$ whose level is less than or equal to $n$, for some $n \in \square$. Let $c_{(n)}$

35 Definitions and notation are borrowed from Brightwell et al. 2003, 4.

36 Sure enough, the identification of the $n^{\text {th }}$ level in a causet with the, say, $n^{\text {th }}$ "stage of Becoming" in that causet's growth is somewhat arbitrary. However, not much hinges on this identification. The argument goes through as long as, for any causet $c \in \Omega$, what is taken to be the part of $c$ that has already become as of any given stage has infinite complement with respect to $c$. Note, in addition, that there is no claim here that the levels of a causet approximated by a general relativistic spacetime will be mapped onto time slices of the embedding spacetime which correspond to sharp values of some global time function. 
denote the thus demarcated subset of $c$ - namely, the subset of all $x \in c$ with the property that every chain ending at $x$ has length at most $n$ (i.e., has at most $n+$ 1 elements):

$$
\max \left\{k \in \square: \exists x_{0}, x_{1}, \ldots, x_{k} \in c \text { with } x_{0} \prec x_{1} \prec \ldots \prec x_{k} \text { and } x_{k}=x\right\} \leq n .
$$

Intuitively, $c_{(n)}$ is supposed to represent past and present as of a given stage of the growth of $c$, i.e., the cumulative accretion of events, or Broad's "sum total of existence", up to that stage.

Recall now that in causal set cosmology based on a CSG dynamics, for almost every world $c$ (viz. for every $c \in \Omega \backslash \Theta$ ), the only propositions of physical significance that may be asserted about $c$ can be analyzed into propositions of the form ' $c \in \operatorname{stem}(b)$ ', $b \in \Omega(\square)$. Moreover, no physical facts can distinguish between isomorphic causet worlds. Accordingly, on the above approach to expounding Choice 1, the necessary condition $[\mathrm{N}]$ is transcribed thus: there exist $b \in \Omega(\square)$ and $n \in \square$ such that for every $c, c^{\prime} \in \Omega \backslash \Theta$, if $c \in \operatorname{stem}(b)$ but $c^{\prime} \notin \operatorname{stem}(b)$, then $c_{(n)}$ is not isomorphic to $c^{\prime}{ }_{(n)}$. But, clearly, this is false! A stem that has not appeared as of any given stage may appear later on and, consequently, for every $b \in \Omega(\square)$ and every $n \in \square$ there exist $c, c^{\prime} \in \Omega \backslash \Theta$ such that $c \in \operatorname{stem}(b)$ and $c^{\prime} \notin \operatorname{stem}(b)$ and $c_{(n)}$ is isomorphic to $c_{(n)}^{\prime}$.

The philosophical moral to be drawn is that, on this approach, causal set cosmology does not support a notion of Becoming consonant with a growing block model of the universe, since it does not bestow physical significance to any proposition referring exclusively to what might count as past and present. The crux of the issue is this, as the proponents of the CSP have acknowledged. ${ }^{37}$ For a stochastic process that takes place against a non-dynamical temporal background, the predicates corresponding to measurable sets can be thought of as logical combinations of simpler predicates whose attribution can be decided in finite time. In the case of the stochastic growth of causal sets, this is true for the cylinder sets, which, however, are devoid of physical significance as they are not covariant. The closest covariant surrogates for the cylinder sets are the stem sets. But the attribution of the predicate corresponding to a stem set, even though it may be verified in finite time, it can be strictly falsified only in the limit of infinite number of stages of growth.

So let us turn to Choice 2. According to it, the temporary locus of Becoming at each stage of growth of a causet contains exactly one element; while the partial order relation $\preceq$ between causet elements admits the interpretation conferred by the locution ' $x$ has already become (is already definite) as of $y$ '. The relation $\preceq$ possesses the formal properties required for this interpretation: it is (i) reflexive ("every event has already become as of itself"), (ii) transitive ("for all events $x, y$ and $z$, if $x$ has already become as of $y$ and $y$ has already become as of $z$, then $x$ has already become as of $z$ "), and (iii) non-universal ("for every event $x$, there is at least one event that has not already become as of $x$ "). ${ }^{38}$

37 Cf. Brightwell et al. 2002, 13-14.

38 For the rationale underpinning these requirements, see also Stein 1991, 148. That $\preceq$ on causets fulfills the requirement of non-universality follows from the trivial fact that every $c \in \Omega$ satisfies the condition: for each $x \in c$ there exists a $y \in c$ such that $y \preceq x$ does not hold. 
It is this conception of Becoming that has been explicitly advocated by some proponents of the CSP. Dowker, for example, in connection with the way in which the RS models can deal with "the problem of Now", has affirmed:

There is growth and change. Things happen! But the general covariance means that the physical order in which they happen is a partial order, not a total order. This doesn't give any physical significance to a universal Now, but rather to events, to a Here-and-Now.

I am not claiming that this picture of accumulating events (which will have to be reassessed in the quantum theory) would explain why we experience time passing, but it is more compatible with our experience than the Block Universe view (Dowker 2005, 458).

And, in a similar vein, she concluded one of her lectures with the following points: ${ }^{39}$

"Becoming" and lack of a global time peacefully co-exist in these models. Things happen, but in a partial order.

In a Sequential Growth Model, the causal past of any newly born element is real: reality accumulates, like sediment, with the events of the past fixed and unchanging and the future as yet unrealised potentiality.

But now there are two qualms one may justifiably have regarding the assertion that the CSP gives new life to the growing block view of the universe. First, this notion of Becoming is not novel: one can trace it in philosophical treatments of "good old" special relativity on Minkowski spacetime. And second, one may worry whether a partial ordering of events together with a solipsist view of each event's present is too weak a base to underpin a robust notion of Becoming that makes absolutely no concessions to eternalist views. I shall bracket here the second kind of concerns as they have been voiced and argued, for and against, in an extensive literature on the metaphysics of special and general relativity. ${ }^{40}$

Let me just go over the basic components of the relevant here conception of Becoming in the context of special relativity. ${ }^{41}$ First, it involves the intention to graft the explication of the terms 'present' and 'temporal Becoming' on the intrinsic geometry of Minkowski spacetime. To this end, Stein (1991) demonstrated that the only plausible two-place relation ' $R x y$ ' between points of Minkowski spacetime that (i) exhibits the necessary formal properties (reflexivity, transitivity, non-universality) to admit the interpretation ' $x$ has already become as of $y$ ', and (ii) is invariant under automorphisms preserving time-orientation, is

39 The referred to talk by Fay Dowker bears the title "Discrete spacetime: Things happen, they just happen in a partial order" and the slides for it are available in the Internet (Dowker 2015).

40 Savitt's (2014) provides an overview and parts II and III of Dieks's (2006) are devoted exactly to the possibility of reconciling temporal Becoming with relativistic spacetime theories.

41 See Stein 1968; 1991; Dieks 2006. 
the relation ' $x$ lies in or on the past light cone of $y$ '. ${ }^{42}$ Second, on this approach, "in Einstein-Minkowski space-time an event's present is constituted by itself alone" (Stein 1968, 15). And, third, the process of Becoming is local, not only in the sense that it reduces to the happenings of individual local events, but also in the sense that the temporal relations in the network of these happenings arise from a partial ordering of non-global nature (Dieks 2006, 173). Becoming is just the successive happening of events along a timelike world line.

It is not my intention to defend (or criticize) this conception of Becoming in relativistic physics. I cannot resist, however, quoting one of Stein's witty remarks to its defense:

At any rate, it is clearly a fact about the historical etymology of our language that the original meaning of the word "present" was not now, but here-now... That remains a current usage: When a soldier at roll call responds "Present!" upon hearing his name, he is not merely announcing that he still exists; he means that he is on the spot (Stein 1991, 159).

Still, my point is that the CSP has not as yet offered a conception of Becoming in physics that is not already familiar from philosophical discussions of relativistic theories on smooth spacetime manifolds. As a last piece of "textual evidence", let me cite an excerpt from Dieks's conclusions:

So the natural view is that the history of our universe is realized by events that come into being; and that they come into being after and before each other as dictated by the partial ordering relation induced by the spacetime structure. According to this proposal the life of the universe is not one linear series of events, but a partially ordered set of events (Dieks 2006, 172-173).

The similarity with Dowker's views cited above is evident.

\section{Conclusion}

The CSP constitutes a vigorous approach towards a quantum theory of gravity that deserves more attention from philosophers than it has attracted so far. And one can argue in support of this claim even irrespectively of one's expectations as to whether the CSP will eventually succeed in "uncovering the Holy Grail", in producing a satisfactory theory of quantum gravity. At any rate, the current state of theoretical physics offers very feeble evidence to ground such expectations, not only for the CSP, but also for programmes that are more popular among physicists and seem to have attained a higher level of maturity, like the string theory programme or the loop quantum gravity programme.

What makes the CSP of particular interest to philosophers is that its development has been accompanied, if not motivated, by the explicitly stated

42 For the exact statement of the result, see the theorem on p. 149 of Stein's (1991). It should be mentioned here that, as Callender and Wüthrich (2014) have shown, causal sets violate what would be an analogue of Stein's theorem, without, however, salvaging a notion of the "present" consistent with Lorentz invariance. 
desire to respond to some challenges with venerable history in the philosophical tradition. ${ }^{43}$ I tried to review and assess the CSP's response to one such philosophical challenge - namely, the challenge to incorporate a dynamic conception of the world, in harmony with a growing block model of the universe, in a (relativistic) physical theory about spacetime.

I argued that the CSP, at its present stage of development, does not meet the challenge for two reasons. First, the only kind of dynamics for causal sets that has been fully elaborated to date, the CSG type of dynamics, cannot be defined with mathematical consistency but in the limit of infinite time "when" causal set growth "has reached completion". This is due to the fact the relevant dynamical law amounts to the specification of the probability measure of a classical stochastic process. Still, this makes the growing block imagery the CSP aspires to flesh out within physics parasitic on a block universe view.

The second reason does not stem from some demand for mathematical consistency but from a physical principle lying at the core of the CSP, discrete general covariance. As I tried to show, this principle deprives the CSP from the conceptual resources to ground truths about the past or the present - resources that are plausibly required of any theory that aims to buttress the metaphysics of past-presentism. The only way out for a proponent of the CSP, who wishes to cling to a notion of Becoming that salvages a dynamic conception of the world, seems to be the stratagem of localizing Becoming and the present. But this move, whether on the right track or not, is not novel: it has been proposed and debated in the context of philosophical attempts to trace a viable notion of Becoming within relativistic spacetime theories on continuous (smooth) manifolds.

As I have mentioned right from the start, both of these obstacles to salvaging a robust notion of Becoming are acknowledged by the proponents of the CSP as well as by philosophers that have commented upon the issue. Why does, then, the CSP continue to retain its appeal vis-à-vis prospects of maintaining some growing block view of the universe? I see only two interrelated reasons for this (other than the obvious one that any metaphysical-ontological interpretations of the theories formulated within the CSP are apt to differ radically once causal sets are endowed with a quantum dynamics). The first has to do with the postulated discreteness of the deep structure of spacetime. In a discrete spacetime, there is not only "before" and "after", but also "previous" and "next": an event does not only have a "past" and a "future", but also a "predecessor" and a "successor". And this "animates" the motion of "now" along a timelike world line. The second reason has been recently pointed out by Callender and Wüthrich (2014): causal sets do exhibit a kind of gauge-invariant growth (measured by the number of elements). But this growth can be taken to graft a notion of temporal Becoming only at the expense of sacrificing various deep-seated intuitions and philosophical convictions. ${ }^{44}$

43 One may adduce, as further reasons, the clarity and parsimony of the CSP's guiding physical principles as well as of its mathematics.

44 For the ramifications, see Callender and Wüthrich 2014. 


\section{References}

Arntzenius, Frank, and Tim Maudlin. 2013. "Time Travel and Modern Physics." In The Stanford Encyclopedia of Philosophy (Winter 2013 Edition), edited by Edward N. Zalta. Stanford: Metaphysics Research Lab, Center for the Study of Language and Information, Stanford University. https://plato.stanford.edu/ archives/win2013/entries/time-travel-phys

Billingsley, Patrick. 1995. Probability and Measure. $3^{\text {rd }}$ edition. New York: John Wiley \& Sons.

Bombelli, Luca, Joohan Lee, David Meyer, and Rafael D. Sorkin. 1987. "Spacetime as a Causal Set." Physical Review Letters 59(5): 521-524. doi: 10.1103/ PhysRevLett.59.521

Broad, C. D. 1923. Scientific Thought. New York: Harcourt, Brace \& Co.

Brightwell, Graham, Fay Dowker, Raquel S. García, Joe Henson, and Rafael D. Sorkin. 2002. "General Covariance and the 'Problem of Time' in a Discrete Cosmology." In Correlations. Proceedings of the Alternative Natural Philosophy Association 23 Conference (August 16-21, 2001, Cambridge, England), edited by Keith G. Bowden, 1-17. London: ANPA.

Brightwell, Graham, Fay Dowker, Raquel S. García, Joe Henson, and Rafael D. Sorkin. 2003 “'Observables' in Causal Set Cosmology." Physical Review D 67: 084031-1-8. doi: 10.1103/PhysRevD.67.084031

Butterfield, Jeremy. 2007. "Stochastic Einstein Causality Revisited." British Journal for the Philosophy of Science 58(4): 805-867. doi: 10.1093/bjps/axm034

Callender, Craig, and Christian Wüthrich. 2014. "What Becomes of a Causal Set." Manuscript.

Crisp, Thomas M. 2003. "Presentism." In The Oxford Handbook of Metaphysics, edited by Michael J. Loux and Dean W. Zimmerman, 211-245. Oxford: Oxford University Press.

Dieks, Dennis. 2006. "Becoming, Relativity and Locality." In The Ontology of Spacetime. Volume 1, edited by Dennis Dieks, 157-176. Amsterdam: Elsevier.

Dieks, Dennis, ed. 2006. The Ontology of Spacetime. Volume 1. Amsterdam: Elsevier.

Dowker, Fay. 2005. "Causal Sets and the Deep Structure of Spacetime." In 100 Years of Relativity - Spacetime Structure: Einstein and Beyond, edited by Abhay Ashtekar, 445-464. New York: World Scientific.

Dowker, Fay. 2006. "Causal Sets as Discrete Spacetime." Contemporary Physics 47(1): 1-9. doi: 10.1080/17445760500356833

Dowker, Fey. 2015. "Discrete Spacetime: Things Happen, They just Happen in a Partial Order." Philosophy and the Sciences at the University of Manchester, May 18. http://www.cicada.manchester.ac.uk/events/workshops/graphsasynchronous-systems/slides-2.pdf

Dowker, Fay, and Sumati Surya. 2006. "Observables in Extended Percolation Models of Causal Set Cosmology." Classical and Quantum Gravity 23(4): 1381-1390. doi: 10.1088/0264-9381/23/4/018 
Earman, John. 1995. Bangs, Crunches, Whimpers, and Shrieks: Singularities and Acausalities in Relativistic Spacetimes. Oxford: Oxford University Press.

Earman, John. 2008. "Reassessing the Prospects for a Growing Block Model of the Universe." International Studies in the Philosophy of Science 22(2): 135164. doi: 10.1080/02698590802496680

Grünbaum, Adolf. 1973. Philosophical Problems of Space and Time. $2^{\text {nd }}$ enlarged edition. Dordrecht: D. Reidel.

Hawking, Stephen W., and George F. R. Ellis. [1973] 1989. The Large Scale Structure of Space-Time. $8^{\text {th }}$ reprint. Cambridge: Cambridge University Press.

Hitchcock, Christopher. 2001. "The Intransitivity of Causation Revealed in Equations and Graphs." Journal of Philosophy 98(6): 273-299. doi: jphil200198614

Lewis, David. 2001. “Truthmaking and Difference-making." Noûs 35(4): 602615. doi: 10.1111/0029-4624.00354

Malament, David B. 1977. “The Class of Continuous Timelike Curves Determines the Topology of Spacetime." Journal of Mathematical Physics 18(7): 13991404. doi: $10.1063 / 1.523436$

Malament, David B. 2007. "Classical Relativity Theory." In Philosophy of Physics, edited by Jeremy Butterfield and John Earman, 229-273. Part A. Amsterdam: Elsevier.

Psillos, Stathis. 2007. "What Is Causation?” In Episteme Reviews: Research Trends in Science, Technology and Mathematics Education, edited by Beena Choksi and Chitra Natarajan, 11-29. Bangalore: Macmillan India.

Rea, Michael C. 2003. "Four-dimensionalism." In The Oxford Handbook of Metaphysics, edited by Michael J. Loux and Dean W. Zimmerman, 246-280. Oxford: Oxford University Press.

Rideout, David P., and Rafael D. Sorkin. 1999. "Classical Sequential Growth Dynamics for Causal Sets." Physical Review D 61: 024002-1-16. doi: 10.1103/ PhysRevD.61.024002

Savitt, Steven. 2002. "On Absolute Becoming and the Myth of Passage." In .), Time, Reality and Experience (Royal Institute of Philosophy Supplement 50), edited by Craig Callender, 153-167. Cambridge: Cambridge University Press.

Savitt, Steven. 2014. "Being and Becoming in Modern Physics." In The Stanford Encyclopedia of Philosophy (Summer 2014 Edition), edited by Edward N. Zalta. Stanford: Metaphysics Research Lab, Center for the Study of Language and Information, Stanford University. https://plato.stanford.edu/ archives/sum2014/entries/spacetime-bebecome

Sorkin, Rafael D. 2005. "Causal Sets: Discrete Gravity." In Lectures on Quantum Gravity (Proceedings of the Valdivia Summer School), edited by Andres Gomberoff and Donald Marolf, 305-327. New York: Plenum.

Sorkin, Rafael D. 2007. "Relativity Theory Does Not Imply that the Future Already Exists: A Counterexample." In .), Relativity and the Dimensionality of the World, edited by Vesselin Petkov, 153-161. Berlin: Springer. 
Stachel, John. 2006. "Structure, Individuality, and Quantum Gravity." In The Structural Foundations of Quantum Gravity, edited by Dean Rickles, Steven French, and Juha Saatsi, 53-82. Oxford: Oxford University Press.

Stein, Howard. 1968. “On Einstein-Minkowski Space-time." The Journal of Philosophy 65(1): 5-23. doi: 10.2307/2024512

Stein, Howard. 1991. "On Relativity Theory and Openness of the Future." Philosophy of Science 58(2): 147-167. doi: 10.1086/289609

Tooley, Michael H. 1997. Time, Tense, and Causation. Oxford: Clarendon Press.

Varadarajan, Madhavan, and David Rideout. 2006. "General Solution for Classical Sequential Growth Dynamics of Causal Sets." Physical Review D 73: 104021-1-10. doi: 10.1103/PhysRevD.73.104021

Wald, Robert M. 1984. General Relativity. Chicago and London: The University of Chicago Press.

Wallden, Petros. 2010. "Causal Sets: Quantum Gravity from a Fundamentally Discrete Spacetime." Journal of Physics: Conference Series 222(1): 012053. doi: 10.1088/1742-6596/222/1/012053

Williams, Donald C. [1951] 1967. "The Myth of Passage." In .), The Philosophy of Time: A Collection of Essays, edited by Richard M. Gale, 98-116. Garden City, New York: Doubleday and Company, Inc.

Williams, Donald. C. 1965. "Physics and Flux: Comment on Professor Čapek's Essay." In Boston Studies in the Philosophy of Science, Vol. II: In honor of Philipp Frank (Proceedings of the Boston Colloquium for the Philosophy of Science), edited by Robert S. Cohen and Marx W. Wartofsky, 464-475. New York: Humanities Press.

Wüthrich, Christian. 2012. “The Structure of Causal Sets." Journal for General Philosophy of Science 43: 223-241. doi: 10.1007/s10838-012-9205-1 